\title{
A Cascade Phase Sensitive Detector- Phase Response
}

\author{
KENZO WATANABE, MEMBER, IEEE, MOHAMMAD MADIHIAN, STUDENT MEMBER, IEEE,
} AND TATSUO YAMAMOTO, MEMBER, IEEE

\begin{abstract}
A phase sensitive detector (PSD) described permits the realization of any phase response by means of many like stages in cascade or in parallel. Two such configurations - one providing the best phase selectivity and the other the best phase discrimination sensitivity - are discussed in detail to demonstrate practical applications. The performance of a prototype detector supports the principles of operation.
\end{abstract}

\section{INTRODUCTION}

T HE CAPABILITY of improving a signal-to-noise ratio (SNR) makes a phase sensitive detector (PSD) very attractive to a high sensitivity detection. This has caused the divergent works on it, leading to the development of various types of PSD [1], such as the lock-in amplifier in a scientific instrument [2], [3], the synchronous or coherent detector in a communication system [4], and the homodyne system in a microwave spectrometer [5], [6]. Though differing in their construction, they are based on essentially the same principle of detection; multiplication of a received signal by the phase-coherent reference carrier. Their phase response is therefore expressible as $\cos \theta$ with $\theta$ being a phase difference between the signal and the reference carrier.

This phase response is not as sensitive as one might expect from their name, but is only available to reject the noise in quadrature with the signal. This results in the highest possible improvement in SNR to be $3 \mathrm{~dB}$ for a white noise [7]. Further improvement is thus only possible by sensitizing the phase response.

An iterative intermediate frequency (IF) amplification by means of like stages in cascade provides the frequency selectivity much higher than that which would be obtained by any individual stage. Likewise, the iterative phase sensitive detection is expected to give the sensitive phase response. This is, however, not possible for a conventional PSD because it cannot provide the phase information indispensable to the subsequent phase sensitive detection. ${ }^{1}$ Expressing the last statement in positive form, we obtain the essential condition for a PSD to be cascadable; it should produce, besides the signal detected phase sensitively, the

Manuscript received May 8, 1978.

- The authors are with the Research Institute of Electronics, Shizuoka University, Hamamatsu 432, Japan.

1 The phase-coherent degenerate parametric amplifier, a special type of PSD, provides the phase information in the phase term of the amplified signal, but in the form too different from the original information to be available for the subsequent phase sensitive detection or amplification [8]. reference carrier that conveys the original phase information.

Based on the above consideration, we have developed a novel scheme for implementing a cascadable PSD. This paper describes the principles of operation, the phase response, and the performance of a prototype detection system.

\section{Principles of Operation}

\section{A. Single Stage PSD}

Fig. 1 shows a schematic diagram of a cascadable PSD. It consists of three main parts; the oscillator driving the frequency translator and the balanced modulator, the heterodyne and homodyne systems forming the reference and signal channels, respectively. The oscillator is referred to as a modulation source when involved in the frequency translator and as a subcarrier source in the homodyne system [1] Here it is identified as a local oscillator and its frequency as an IF.

The heterodyne system consists of a phase shifter, frequency translator, and mixer 1. It operates as a frequency converter. The homodyne system consists of a phase shifter, balanced modulator, and mixer 2 . It operates as a phase detector.

Now let us assume that the phase-coherent reference carrier and the signal are written to be of the form

$$
\begin{aligned}
& v_{r(i-1)}=V_{r(i-1)} \cos \left(\omega_{i-1} t+\theta_{i-1}\right) \\
& v_{s(i-1)}=V_{s(i-1)} \cos \omega_{i-1} t
\end{aligned}
$$

respectively. After passing through the phase shifter, the reference carrier is divided into two parts: one applied directly to mixer 2 and the other to the frequency translator. The output of the frequency translator is then given by

$$
v_{T}=V_{r(i-1)} \cos \left\{\left(\omega_{i-1}+\omega_{i}\right) t+\theta_{i-1}+\phi_{i}\right\} .
$$

This output is mixed with the phase-shifted signal in mixer 1 to provide the reference output

$$
v_{r(i)}=V_{r(i)} \cos \left(\omega_{i} t+\theta_{i}\right)
$$

where

$$
\begin{aligned}
V_{r(i)} & =G_{i} V_{r(i-1)} V_{s(i-1)} \\
\theta_{i} & =\theta_{i-1}-\psi_{i}+\phi_{i}
\end{aligned}
$$




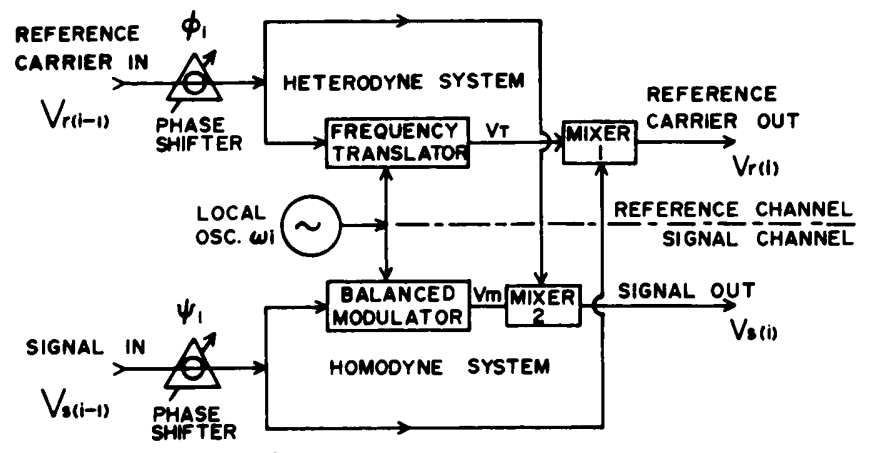

Fig. 1. A single stage PSD.

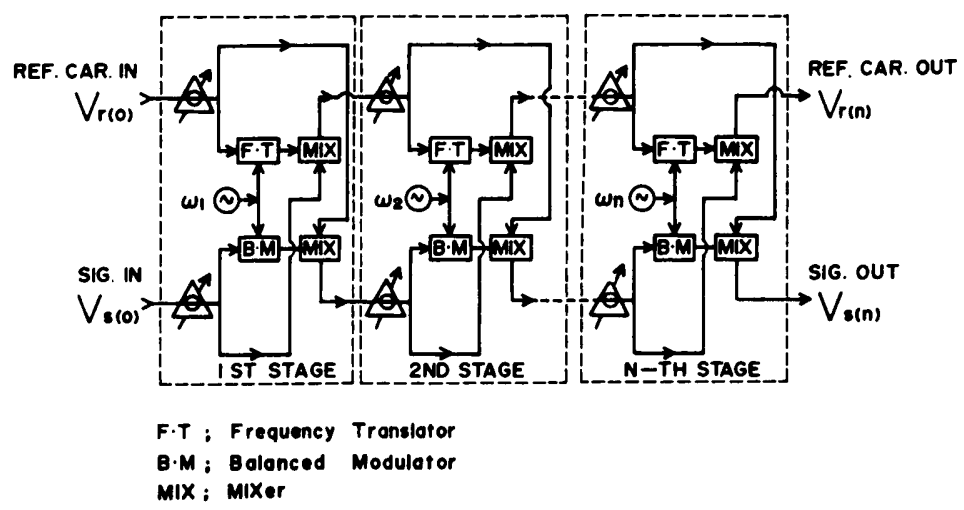

Fig. 2. A multistage PSD.

and $G_{i}$ represents the conversion gain of the heterodyne system. In (4), only $\omega_{i}$ component is retained because of the implicit assumption of the bandpass filter in the mixer. by

Similarly, the output of the balanced modulator is given

$$
v_{m}=V_{s(i-1)} \cos \left\{\left(\omega_{i-1} \pm \omega_{i}\right) t+\psi_{i}\right\} .
$$

Being mixed with the phase-shifted reference carrier in mixer 2 , it provides the signal output:

where

$$
v_{s(i)}=V_{s(i)} \cos \omega_{i} t
$$

$$
V_{s(i)}=H_{i} V_{r(i-1)} V_{s(i-1)} \cos \theta_{i}
$$

and $H_{i}$ is the conversion gain of the homodyne system. Also, only $\omega_{i}$ component is retained in (8).

Equations (5) and (9) assume the square law operation of the mixer. When the amplitude of the reference carrier $V_{r(i-1)}$ is large enough to assure the mixer of a linear law operation, (5) and (9) become independent of $V_{r(i-1)}$, reducing to

$$
V_{r(i)}=G_{i} V_{s(i-1)} \quad V_{s(i)}=H_{i} V_{s(i-1)} \cos \theta_{i}
$$

The phase response given by $(9)$ or (10) is the same as that of a conventional PSD. It should be noticed, however, that the reference and signal outputs take the same form as that of each input, thus retaining the phase information. It is this retained information that makes the iterative phase sensitive detection possible.

\section{B. Multistage PSD}

Taking the outputs of single stage PSD for the inputs to a subsequent PSD, one can cascade it, as shown in Fig. 2, to constitute the multistage PSD. The outputs of $n$th stage are

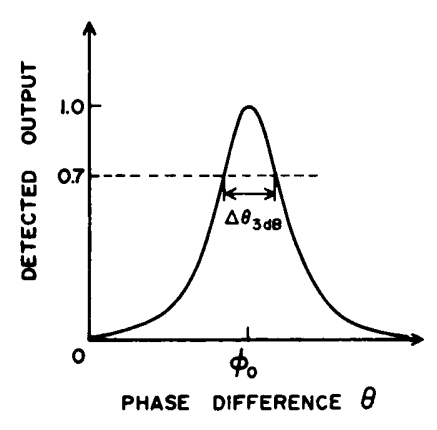

Fig. 3. The phase selective response.

given by (4) and (8) with the subscript $i$ replaced by $n$. Lowering order $n$ progressively using (5) and (9) for the square law and (10) for the linear law operation, one can express the outputs of $n$th stage in terms of the signal amplitude and the original phase information. For square law operation,

$$
\begin{aligned}
V_{r(n)}= & G V_{r} \prod_{i=2}^{n} \cos ^{2 n-i}\left\{\theta+\sum_{j=1}^{i-1}\left(\phi_{j}-\psi_{j}\right)\right\} \\
V_{s(n)}= & H V_{s} \cos \left\{\theta+\sum_{i=1}^{n}\left(\phi_{i}-\psi_{i}\right)\right\} \prod_{i=2}^{n} \cos ^{2 n-1} \\
& \cdot\left\{\theta+\sum_{j=1}^{i-1}\left(\phi_{j}-\psi_{j}\right)\right\}
\end{aligned}
$$

and for linear law operation,

$$
\begin{aligned}
& V_{r(n)}=G V_{r} \prod_{i=1}^{n-1} \cos \left\{\theta+\sum_{j=1}^{i}\left(\phi_{j}-\psi_{j}\right)\right\} \\
& V_{s(n)}=H V_{s} \prod_{i=1}^{n} \cos \left\{\theta+\sum_{j=1}^{i}\left(\phi_{j}-\psi_{j}\right)\right\}
\end{aligned}
$$

where $G$ and $H$ represent the overall gain of the reference and signal channels, respectively, and $\theta$ is the phase difference between the original signal and the reference carrier.

\section{Phase Response}

In what follows every stage is assumed to be in linear law operation. The description is, however, equally applicable to the square law operation. Various phase responses are possible by changing $n, \phi_{i}$, and $\psi_{i}$. Two of them are important from the practical point of view: the phase selective and the discrimination responses.

\section{A. Phase Selective Response}

The best selective response is obtained when $\phi_{i}=\psi_{i}$ $(i=2,3, \cdots n)$. The response then reduces to

$$
V_{s(n)}=H V_{s} \cos ^{n}\left(\theta-\phi_{0}\right)
$$

where

$$
\phi_{0}=\psi_{1}-\phi_{1} .
$$

This is depicted in normalized form in Fig. 3. Just as the half bandwidth measures the frequency selectivity of a bandpass 


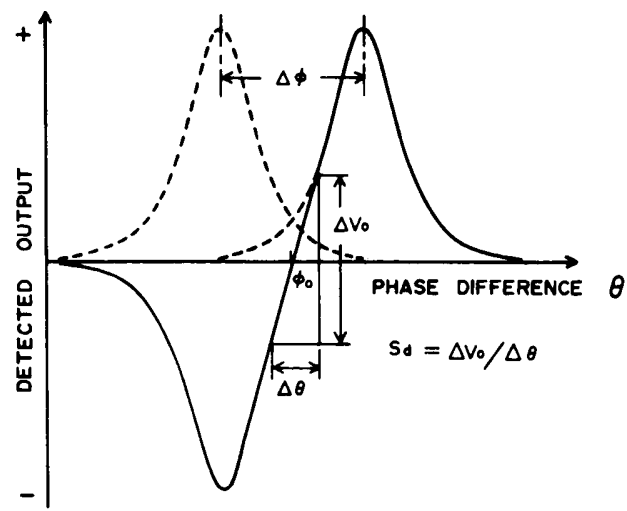

Fig. 4. The phase discrimination response (solid curve) given by the difference between two phase selective responses (dotted curves).

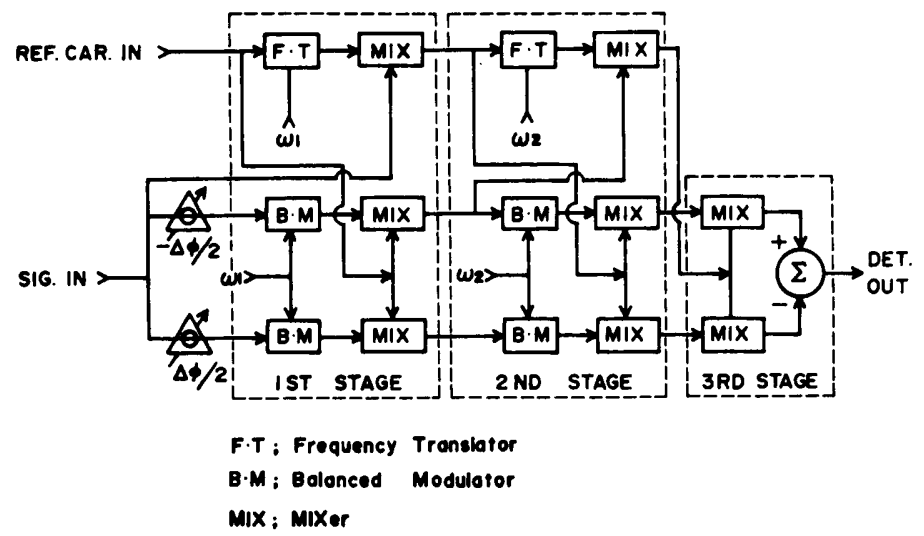

Fig. 5. A schematic diagram of three stage phase discriminator.

filter, the half phase-bandwidth measures the phase selectivity. From (15), it is given by

$$
\Delta \theta_{3-\mathrm{dB}}=2 \cos ^{-1} 2^{-(1 / 2 n)} .
$$

Incidentally, $\Delta \theta_{3-\mathrm{dB}}$ is $\pi / 2 \mathrm{rad}$ for $n=1$, and $0.2 \pi \mathrm{rad}$ for $n=7$. This phase response is useful for detecting a signal with the fixed frequency and fixed phase.

\section{B. Phase Discrimination Response}

The phase discrimination response takes the differential form of the phase selective response. The differential operation is easily accomplished in a conventional PSD by shifting the reference phase by $\pi / 2 \mathrm{rad}$. This is, however, not true in the present PSD as evidenced by shifting $\phi_{0}$ in (15) by $\pi / 2$. Hence the differential operation should be replaced by the difference one. Fig. 4 explains schematically how this is operated; the difference between the two selective responses symmetrical about $\phi_{0}$ gives the phase discrimination response when their spacing $\Delta \phi$ is appropriate.

The discrimination sensitivity, defined as the ratio of an incremental change in output voltage to an incremental change in phase, is given by

$$
S_{d}=\Delta V_{0} / \Delta \theta=2 n \sin (\Delta \phi / 2) \cos ^{n-1}(\Delta \phi / 2)
$$

and becomes maximum when $\Delta \phi / 2=\tan ^{-1}(n-1)^{-(1 / 2)}$. Incidentally, when $n=3, S_{d}$ takes the maximum value 2.3 when $\Delta \phi$ is $70^{\circ}$, which is 2.3 times larger than that of a conventional PSD. One scheme for implementing the phase discriminator quoted herein is shown in Fig. 5.

This phase response is useful for demodulating a phase information.

\section{Prototype Detector}

A prototype detection system has been implemented for experimental confirmation of the principles described above. It consists of seven stages of PSD, each individual stage except for the last one having the same configuration as that shown in Fig. 1. The last stage comprises only one mixer followed by a low-pass filter, thus providing not IF but video output. The signal frequency is chosen to $9.5 \mathrm{GHz}$. This choice facilitates the phase measurement because of a commercially available calibrated phase shifter. The IF's are $47 \mathrm{MHz}, 30 \mathrm{MHz}, 10.7 \mathrm{MHz}, 3.2 \mathrm{MHz}, 1 \mathrm{MHz}$, and $450 \mathrm{kHz}$. These are so chosen as to avoid the crosstalk between the harmonics.

The frequency translator in each stage comprises a balanced modulator followed by a bandpass filter. The configurations of the heterodyne and homodyne systems are thus identical except for the bandpass filter.

The balanced modulator in the microwave stage consists of a circulator and a p-i-n diode mounted $\lambda_{g} / 4$ away from a short. Those in IF stages are commercial double-balanced mixers available in the form of integrated circuit (IC), which facilitates the implementation of the system. The same IC's are also used as the mixers involved in each IF stage. The bandpass filter in the microwave stage is a transmission-type cavity, and those in IF stages are conventional IF amplifiers. They are tuned to one of the sidebands generated by the preceding balanced modulator, thereby playing the role of the frequency translator in combination with the balanced modulator.

The phase shifts in each stage, $\phi_{i}$ and $\psi_{i}$ in Fig. 2 , are set to be equal. Therefore, every stage retains the phase difference between the original signal and reference carrier. This simplifies the phase response of the whole system, giving the best phase selectivity.

The signal power is held fixed to $-40 \mathrm{dBm}$. With this power, each IF stage delivers the reference output of $1.0 \mathrm{~V}_{\mathrm{p}-\mathrm{p}}$ at its maximum. This value is large enough to assure the mixer of a linear law operation.

The measurement was made by varying the signal phase by means of a calibrated phase shifter. The detected outputs, normalized to its maximum value, are plotted in Fig. 6 . The abscissa is referenced to the reading of the phase shifter when the maximum output is obtained. For comparison two curves are drawn showing $\cos ^{7} \theta$ and $\cos ^{11} \theta$.

By inspection it is found that the data for $\theta$ close to zero fit well the curve of $\cos ^{7} \theta$. This response is just what we can expect from the multistage PSD in linear law operation. As the phase difference $\theta$ increases, the data deviate from this curve and approach the curve of $\cos ^{11} \theta$. This is explained as follows: In the present configuration, the reference output as well as the signal output decreases with increasing $\theta$. This reduction is more serious to the later stage, forcing the mixers involved to operate in not the linear but the square 


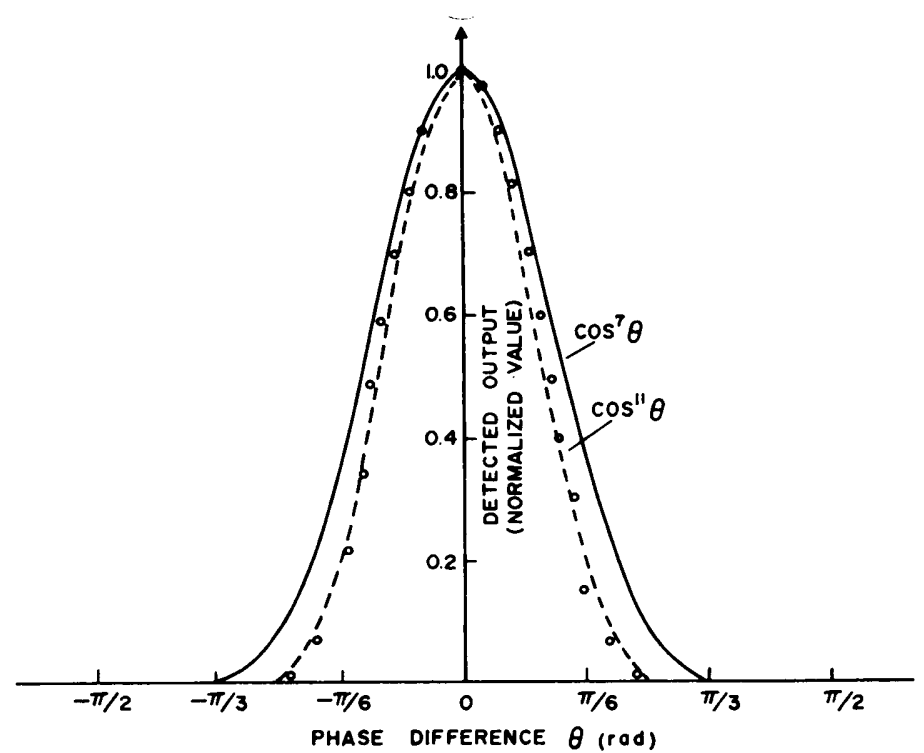

Fig. 6. The phase selective response of a prototype detector consisting of seven stages of PSD in cascade.

law region. Assuming incidentally that for $\theta$ close to $\pi / 6$, the last four stages are forced to operate in the square law region, one obtains the phase response of $\cos ^{11} \theta$. By the way, the reference output of $0.85 \mathrm{~V}_{\mathrm{p}-\mathrm{p}}$ is necessary to assure the mixer of linear law operation, whereas reference output of third stage is $0.65 \mathrm{~V}_{\mathrm{p}-\mathrm{p}}$ when $\theta=\pi / 6$. The half phasebandwidth $\Delta \theta_{3-\mathrm{dB}}$ measured is $0.18 \pi \mathrm{rad}$, in good agreement with the value $0.2 \pi$ rad quoted in Section III-A.

\section{Conclusions}

The circuit configuration and principles of operation of a cascadable PSD have been described. The proposed PSD features its capability of providing any phase response by being connected in cascade or in parallel. Particular atten- tion has been paid to the phase selective and discrimination responses from the practical viewpoint. The prototype detector has provided the sensitive selective response, supporting the principles.

The PSD described here is applicable to a lock-in amplifier to improve the phase selectivity and also to the phase comparison detector to demodulate phase-shift keying signals. The sensitized phase response realized here is considered to bring about a significant improvement in SNR, which is now under way.

\section{ACKNOWLEDGMENT}

The authors are much indebted to M. Ashiki for his help in the implementation and the measurement of the prototype detector.

\section{REFERENCES}

[1] J. D. Dyson, "The measurement of phase at UHF and microwave frequencies," IEEE Trans. Microwave Theory Tech., vol. MTT-14, pp. 410-423, Sept. 1966.

[2] J. T. Lue, "Junction impedance measurements of diodes by a simplified lock-in amplifier," IEEE Trans. Instrum. Meas., vol. IM-26, pp. 415-419, Dec. 1977.

[3] D. P. Blair and P. H. Sydenham, "Phase sensitive detection as a means to recover signals buried in noise," J. Phys. E: J. Sci. Instrum., vol. 8, pp. 621-627, Aug. 1975.

[4] A. B. Carlson, Communication Systems. New York: McGraw-Hill, 1965, pp. 206-235.

[5] H. C. Praddaude, " $100 \mathrm{kHz}$ homodyne, $35 \mathrm{GHz}$ reflection-cavity spectrometer with phase-lock of the reference signal and low frequency field modulation," Rev. Sci. Instrum., vol. 38, pp. 339-347, Mar. 1967.

[6] D. L. Jaggard and R. J. King, "Sensitivity and dynamic range considerations for homodyne detection systems," IEEE Trans. Instrum. Meas., vol. IM-22, pp. 331-338, Dec. 1973; also R. J. King, Microwave Homodyne Systems. London, England: Peter Peregrinus Ltd., 1977.

[7] K. Watanabe and I. Takao, "Optimum modulator design for a high sensitivity homodyne system-Binary modulation method," IEEE Trans. Microwave Theory Tech., vol. MTT-23, pp. 354-359, Apr. 1975.

[8] L. A. Blackwell and K. L. Kotzebue, Semiconductor-Diode Parametric Amplifiers. Englewood Cliffs, NJ: Prentice-Hall, 1961, pp. 72-79. 